\title{
Mobile Agent Based Publication Alerting System
}

\author{
Ozgur Koray Sahingoz ${ }^{1}$ and A. Coskun Sonmez ${ }^{2}$ \\ 1 Air Force Academy, Computer Engineering Department, Yesilyurt, Istanbul, Turkey \\ sahingoz@hho.edu.tr \\ ${ }^{2}$ Yildiz Technical University, Computer Engineering Department, Yildiz, 34349, \\ Istanbul, Turkey \\ acsonmez@ce.yildiz.edu.tr
}

\begin{abstract}
This paper introduces a distributed publication alerting service which increases the amount of information in notification message while using information hiding principle. It is aimed to design a mobile agent based publication alerting system (MABPAS) which uses mobile agents to dispatch notification information (generally all text information) about produced publication to subscribers. MABPAS combines the advantages of publish/subscribe communication model and mobile agents into a flexible and extensible distributed execution environment.
\end{abstract}

\section{Introduction}

Publication alert is an interactive feature that makes it possible for authors and publishers to directly post notification to an information dissemination system (like mail groups or dispatching services) about recently published works. This approach makes subscribers of the system aware of recent publications. To alert more subscribers it is necessary to put more information to notification message.

Publication alerting systems are used to provide online platform for keeping subscribers to be informed about any publication in a new issue of a journal, magazine, newsletter or proceedings. Publications can be either scientific publications like technical reports, conference papers, journal papers, books or social publications like novels, adventure books and magazines. In most of the previous works, notifications about publications are performed by mailing lists with limited expressiveness and functionality. To improve the efficiency of the alerting system we developed a mobile agent based publication alerting service (MABPAS) which takes advantage of mobile agents to dispatch necessary information (generally all text information) about produced publication to subscribers. MABPAS uses the execution environment of an agent based distributed event system [1] which uses mobile agents as event messages (called as agvent(agent event)).

\section{Publication Alerting System}

MABPAS implements the publish/subscribe protocol, thus enabling many-to-many interaction of loosely coupled entities. It also allows publishers and subscribers to 
dynamically connect and disconnect from the system, a capability that extends the flexibility of the working environment. Two properties of MABPAS make it distinctive from other alerting systems:

- Autonomy: the agent structure of the publication agvents represents both the capabilities (ability to compute something, to decide target nodes) and the preferences over how these capabilities can be used. Thus, publication agvents have the ability to reason about how they use their resources and selects target subscribers by using knowledgebase of the brokers. These brokers are called as agvent servers whose main role is providing an execution platform for publication agvents.

- Conversation-Negotiation: since the agvents are autonomous, they must negotiate with other stationary agents to gain access to their resources and capabilities. It is important to hide information in the agvent structure; therefore both agvent servers(ASs) and subscribers cannot see information in it. At the same time, agvents cannot reach directly to the resources of ASs. Agvents can obtain necessary information for their routing operations by talking with stationary agent of an AS. Communication with the subscriber is a little bit different. There is no forwarding operation after arriving on a subscriber. Therefore a publication agvent does not need to talk with the subscriber's stationary agent. However a subscriber agent has to communicate with the incoming publication agvent to get necessary information. To accomplish this subscriber agent follows a "conversation sequence", where multiple messages are exchanged according to some pre-defined protocols.

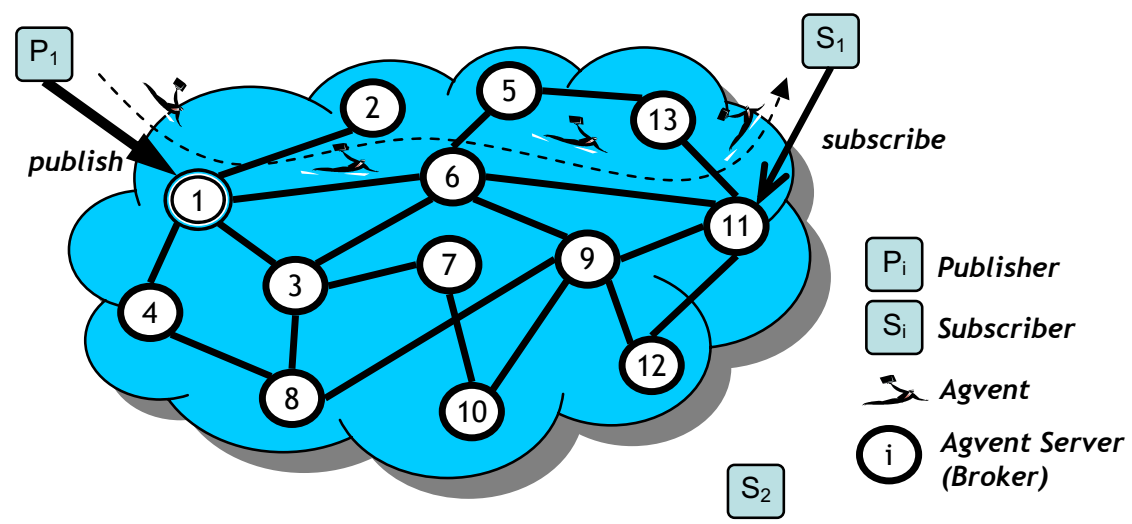

Fig. 1. Dispatching Mechanism of MABPAS

\section{MABPAS consists of four main components as depicted in Figure 1;}

1. Publishers are components which produce publications and publish their relevant agvents to the alerting system (i.e. publishing houses or singular authors).

2. Subscribers are the consumers of the system like libraries, researchers, academic staff or students. They issue subscriptions/profiles that describe the publications/ agvents they want to receive.

3. Agvents are created by publishers according to relevant publications and they are the main components of the system. A publication agvent is a collection of code and 
data (generally all text information of the publication) that migrates through the dispatch service. Dispatch service is comprised of a network of distributed nodes and an agvent routes itself at each node on the path towards its target subscribers. The agvent structure of MABPAS is named as PublicationAgvent which contains attributes and behaviors of the relevant publication. These properties are used for establishing an efficient filtering mechanism. PublicationAgvent is a class definition which contains not only the filterable attributes like publisher_name, publish_date, pages, authors, publication_name, list_price...etc but also filterable methods like ReferenceContains(String AuthorName), TOCContains(String topic), WholesalePrice (int amount,String destination)...etc. The filtering process of the system is the keystone of the alerting service. System enables subscribers to subscribe on both the publication agvents' attributes and methods. By using this type filtering mechanism; it is possible to compose complex subscriptions, message traffic of system is reduced, and dispatching of irrelevant messages is prevented.

There are also some methods which are used for conversation with subscribers. These methods are not filterable methods and therefore they are not advertised. These methods are used by publication agvent according to subscribers' requests.

4. Agvent Server processes incoming subscriptions and advertisements according to a protocol which includes their propagation to adjacent/neighbor ASs. Agvents are selfrouting, that is, they are responsible for determining their own paths through the network, utilizing a minimal set of facilities provided by ASs. ASs support incoming agvents by providing a simple, architecturally independent environment for the receipt and execution of publication agvents. An AS has no access to the content of the published agvent, which simplifies its role and consequently facilitates the server development process. Information hiding principle also meets confidentiality of publication information.

\section{Performance Evaluation}

We've selected average publication (agvent) distribution time as an appropriate benchmark for evaluating performance of MABPAS. To calculate this we use a network with properties as shown in Table 1.a and we dispatch a publication agvent with the properties as shown in Table 1.b.

Table 1. Model Parameters for Performance Evaluation

\begin{tabular}{|c|l|}
\hline Properties & \multicolumn{1}{|c|}{ Explanation } \\
\hline LAN & 100 Mbit Eternet \\
\hline LAN Op. Sys & Windows NT \\
\hline & Pentium III, IV, Centrino \\
& $1.0,3.0,1.6 \mathrm{GHz}$ CPU \\
Computer & $512 \mathrm{Mb}$ RAM \\
Properties & 256, 512, 2000 Kb Cache \\
& $133,800,400 \mathrm{MHz}$ FSB \\
& Windows XP Op. Sys. \\
\hline
\end{tabular}

a) Network Properties

\begin{tabular}{|l|c|}
\hline \multicolumn{1}{|c|}{ Properties } & Value Space \\
\hline \# of advertisements & $5.000-15.000$ \\
\hline \# of subscriptions & $10.000-30.000$ \\
\hline \# of neighbor agvent servers & $1-7$ \\
\hline \# of subs. connected to an AS & $5-100$ \\
\hline \# of ASs in dispatching system & 100 \\
\hline \# of attributes in subscription & $0-15$ \\
\hline \# of behaviors in subs. & $0-10$ \\
\hline \# of parameters for method call & $0-5$ \\
\hline
\end{tabular}

b) System Properties 
We define distribution time as the elapsed time from the generation of a publication agvent to its reception by all subscribers that are interested in that agvent type. In our analysis, agvent generation is the submission of a publication agvent to an AS. In our preliminary analysis, we established an objective of determining the efficiency of the AS delivery without incurring concurrency into the system. Our goal was to examine the performance and scalability of the alerting system.

Table 2. Agvent Sizes

\begin{tabular}{|c|c|c|c|}
\hline Type of Publication & \# of Word & \# of Characters & $\begin{array}{c}\text { Approximate } \\
\text { Agvent Size (Kb) }\end{array}$ \\
\hline Article in a Newspaper & 641 & 4301 & 4.60 \\
\hline Article in a Magazine & 1943 & 12658 & 13.93 \\
\hline Paper with 8 page & 3116 & 17260 & 22.35 \\
\hline Paper with 12 page & 7504 & 40660 & 53.81 \\
\hline PHD Thesis & 44650 & 318920 & 320.17 \\
\hline $\begin{array}{c}\text { Symposium Proceedings } \\
\text { With 50 Papers }\end{array}$ & 237300 & 1308750 & $1,700.00$ \\
\hline $\begin{array}{c}\text { Book with 870 Pages } \\
\text { (i.e. Comp. Netw. Tanenbaum) }\end{array}$ & 325070 & 1600654 & $2,330.00$ \\
\hline
\end{tabular}

Dispatching of an agvent is directly related with its size. It changes according to agvent's type and the data it contains. Table 2 shows the change in agvent size relative to number of words in a publication.

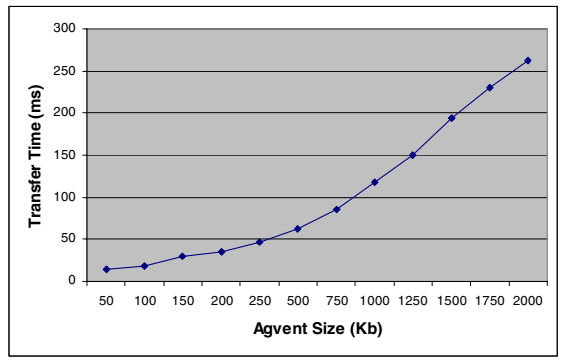

a) Transfer time between two ASs

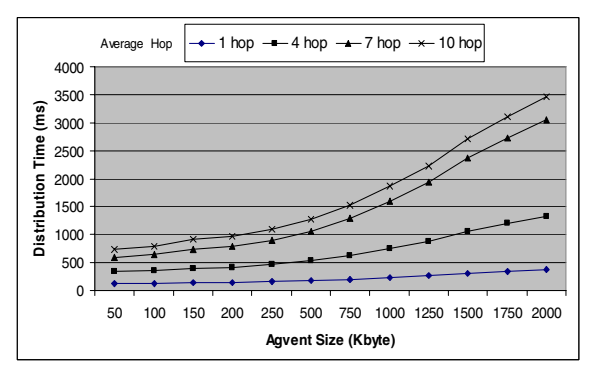

b) Agvent Distribution Time

Fig. 2. Experimental Results for Agvent Transfer Times

To compile realistic network topologies that approximate the behavior of real wide-area networks, we used a generator of random network graphs that implements the Transit-Stub model [2] with 100 agvent servers. We ran a simple benchmark with a single publisher and a multiple number of subscribers, which were distributed evenly on this alerting service. As we were sure that there was no concurrent agvent delivery, we could compute the theoretical best average delivery time. Figure 2.a shows agvent transfer times between two ASs relative to agvent sizes. Figure 2.b shows agvent distribution time in MABPAS with previously mentioned system parameters for different average hops between publishers and subscribers. 


\section{References}

1. Sahingoz, O. K., and Erdogan N.:Agvent:Agent Based Distributed Event System, Proceedings of 30th Conference on Current Trends in Theory and Practice of Computer Science (SOFSEM 2004), Czech Republic, (2004), 144-153.

2. Zegura, E. W., Calvert, K. L., and Bhattacharjee, S.: How to Model an Internetwork. In Proceedings of IEEE INFOCOM '96, San Framcisco CA, U.S.A., (1996), 594-602 